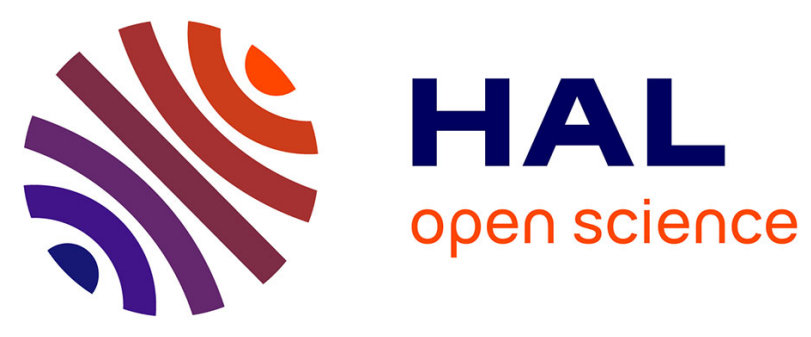

\title{
Reasoning About Knowledge in Context
}

Franck Lihoreau, Manuel Rebuschi

\section{To cite this version:}

Franck Lihoreau, Manuel Rebuschi. Reasoning About Knowledge in Context. Manuel Rebuschi; Martine Batt; Gerhard Heinzmann; Franck Lihoreau; Michel Musiol; Alain Trognon. Interdisciplinary Works in Logic, Epistemology, Psychology and Linguistics, 3, Springer, pp.155-179, 2014, Logic, Argumentation \& Reasoning, 978-3-319-03043-2. 10.1007/978-3-319-03044-9_7 . halshs-01231960

\section{HAL Id: halshs-01231960 \\ https://shs.hal.science/halshs-01231960}

Submitted on 21 Nov 2015

HAL is a multi-disciplinary open access archive for the deposit and dissemination of scientific research documents, whether they are published or not. The documents may come from teaching and research institutions in France or abroad, or from public or private research centers.
L'archive ouverte pluridisciplinaire HAL, est destinée au dépôt et à la diffusion de documents scientifiques de niveau recherche, publiés ou non, émanant des établissements d'enseignement et de recherche français ou étrangers, des laboratoires publics ou privés.

\section{(ㅇ)(1) $\$$}

Distributed under a Creative Commons Attribution - NonCommercial - NoDerivatives 44.0 


\title{
Reasoning About Knowledge In Context
}

\author{
Franck Lihoreau ${ }^{1}$ and Manuel Rebuschi ${ }^{2}$ \\ ${ }^{1}$ Instituto de Filosofia da Linguagem, Universidade Nova de Lisboa, \\ ${ }^{2}$ LHSP - Archives H. Poincaré, MSH Lorraine, Université de Lorraine
}

\begin{abstract}
In this paper we lay out the conceptual and technical foundations of a general framework that will allow us to talk and reason about the connections between knowledge and context. Based on the notion of "contextual models", its static part will make it possible to capture formally, by means of the same language and the same semantics, a number of epistemological positions among the most prominent in the recent philosophical literature on knowledge, and to investigate the logical properties and connections they end up attaching to their respective notions of knowledge and context. This "static" component is augmented with a "dynamic" formalization of context based on a simplified version of Discourse Representation Theory, that will allow us to account for the contribution of epistemic statements to the evolution of the "score" of a conversation about knowledge.
\end{abstract}

\section{Introduction}

A major goal of post-cartesian epistemology is to respond to radical skepticism, the view that we know (almost) nothing of what we ordinarily take ourselves to know. The argument most commonly associated with this view is the so-called "argument from ignorance". It starts with the premise that we cannot exclude the possibility that we might be in such alternative worlds as those described by Descartes' Evil Genius hypothesis or by Putnam's Brain in a Vat scenario. The alleged reason for this inability is that these skeptical hypotheses are designed in such a way that if we were in the skeptical worlds they describe, we'd have exactly the same experiences, memories, beliefs, etc., as those that we actually have, so that for all we know, we might be in these worlds of mass(ive) error. From here, the skeptic's reasoning takes us to the conclusion that we do not know any (or most) of the things that we ordinarily take ourselves to know, for instance, that we have hands, that we are sitting at our desk, etc.

The problem is, of course, that this skeptical conclusion goes against our powerful tendency to think that we do know a lot about many things. In the last decades, emphasis has been put on the importance, in dealing with the problem, of taking (some notion of) context into consideration when thinking about knowledge and knowledge ascription. This shows in the ever-growing discussions of so-called "contextualist" approaches as defended by Cohen [5, 4], DeRose [7, 8, 9], Heller [18, 19], Lewis [26, 27] and others, as well as the increasing interest in theories of "subject-sensitive" knowledge ascriptions $\grave{a}$ la Hawthorne [21] and Stanley [35], and of "assessment-sensitive" ascriptions à la Macfarlane 29], to mention but a few of the available treatments that make context play a significant epistemological role.

In Section 2, we present four major epistemological positions on the problem of skepticism, with an eye to showing the importance of (different notions of) context in the recent philosophical discussions on knowledge. In Section 3, we provide the foundations of a general formal framework based on the technical notion of "contextual models", that will make it possible to capture those epistemological positions by means of the same language and the same semantics, and to investigate the logical connections they are bound to endorse between knowledge and context. Finally, Section 4 adds to that "static" formalism 
a "dynamic" formalization of context based on a simplified version of Discourse Representation Theory, that will allow us to account for the apparent variation over the course of a conversation in the epistemic standards intended to by the participants, and to overcome such difficulties as the logical omniscience problem that usually plagues normal modal logical approaches to knowledge.

In brief, our purpose is to provide formal tools for exploring reasoning about knowledge in context in both its static and dynamic aspects.

\section{Highlights of Informal Epistemology}

In this section we briefly describe and illustrate four prominent positions on the problem of skepticism which, through the discussions they have given rise to in the recent philosophical literature on knowledge, have significantly contributed to making context an issue of prime epistemological importance.

Anti-Skeptical Invariantism The first position, anti-skeptical invarantism as we will call it, which can also be found in the literature under the name "moderate invariantism" or "radical anti-skepticism", has it that many and perhaps most of our ordinary knowledge claims are literally true. It is endorsed by the ordinary language philosopher Austin $[3],{ }^{1}$ who observes that the epistemic standards that the skeptic has in mind and which require from us the ability to exclude absolutely all possibilities of error, including the most far-fetched ones, is very different from those which govern our everyday knowledge claims and which do not have such stringent requirements. In everyday life, the standards in place are such that as long as we have no reason to think, say, that we might be brains in vats, we are not required to rule out this possibility to properly count as knowing, say, that we have hands. Now, according to Austin, the only standards that can be legitimate are those that match our ordinary practices of knowledge ascription. This is the case with those in place in everyday life: we - ordinarily competent speakers judge many and perhaps most of our everyday knowledge claims to be correct. Not so with the skeptic's standards which would make it merely impossible to ever use "know" correctly. So, on this view, we do know a lot.

Skeptical Invariantism The next position, skeptical invariantism as we will call it, or simply skepticism, goes exactly in the opposite direction in claiming that most and perhaps all of our ordinary knowledge claims are literally false. This claim is held by Unger who, in [36] for instance, proposes to relate the word "know" to a class of natural language expressions that are systematically used erroneously, yet in a pragmatically correct way by competent speakers, and which he labels "absolute terms" as their application admits no degree/exception. The predicate "flat" is one such term: (most of) our ordinarily flatness ascriptions are literally false since a surface is flat only if it has absolutely no bump or whatever other irregularity on it, but no real physical surface can meet this condition, be it at the microscopic level. They are, however, close enough to be flat given our everyday life interests, goals, presuppositions, etc., for those ascriptions to be justifiedly made, pragmatically speaking. Likewise, "know" too is an absolute term: because we cannot rule out all logical possibilities of error, (most of) our ordinarily knowledge ascriptions are literally false; yet, we are pragmatically justified in making them in that we are close enough, with respect to our everyday purposes, interests, etc., to satisfying the conditions for a true ascription. In any case, on this view, we know nothing or so.

Contextualism A third position, contextualism as it is called, aims at overcoming the apparent conflict between skepticism and anti-skepticism in holding that the truth or falsity of knowledge ascriptions depends on the context in which they are made. It is defended, among others (Cohen, DeRose, Heller,

\footnotetext{
${ }^{1}$ It is very common in the literature to find this position associated with the name of G. E. Moore, and for this reason called "Moorean invariantism". We find it more convenient to appeal to Austin instead.
} 
etc.), by Lewis $[26,27]$ who claims, that for an ascription of the form " $S$ knows that $p$ " to be true, the possibilities of error that $S$ must be able to rule out are all and only those that are relevant in the context of the ascription, e.g. those attended to by the ascriber. Now, this condition is met when no not- $p$ possibility is contextually relevant. This is the case in everyday contexts for most contingent propositions, e.g. that we have hands, as in these contexts the far-fetched skeptical possibility that we might be handless brains in vats simply is irrelevant, hence need not be ruled out. By contrast, in a philosophical context where such skeptical possibilities have been raised and are being attended to, they are relevant and need be excluded; but we are unable to rule them out. In these contexts it is therefore false that we know that we are not brains in vats and that we have hands. It is true that we know a lot in ordinary contexts, and very little in skeptical contexts. More generally, on the contextualist view, whether it is true that one knows something will depend on the focus, interests, stakes, presuppositions, etc., that make up the context of the "attributor", the person who is attributing/denying knowledge.

Subjectivism On the fourth position too, subjectivism (or sensitive moderate invariantism, or subjectsensitive invariantism as it is often called), as defended by Hawthorne 21] or Stanley [35], such factors as attention, interests, stakes, etc., are considered relevant to the truth of knowledge ascriptions, but only insofar as they make up the context, not of the attributor, but the "subject", the person who is being attributed/denied knowledge. This is clear from Hawthorne who insists that the practical importance, for the subject, of being right and not making a mistake is epistemically crucial, as a subject's anxiety can contribute to making certain possibilities of error salient to him, where salience is equated with relevance. These and only these possibilities will have to be ruled out for the subject to truly count as knowing. Thus, one can know more by worrying less. In particular, "the philosopher who worries about being a brain in a vat, etc., will know less than the dullard who doesn't” (21]: 167).

The formal framework to be described in the next section will help us understand more precisely what the connections between subjectivism, contextualism, anti-skepticism and skepticism are.

\section{Static Formalism}

In this section, we first propose a formal framework for reasoning about knowledge in context, and then show how this framework can be used to capture the various epistemological positions described in the previous section.

\subsection{The Formal Framework}

We first describe the "epistemic language" we will be using throughout the paper, we provide a "contextual semantics" for it that allows for four possible definitions of truth for epistemic formulas, and we investigate what and how usual logical properties of knowledge are preserved in our proposed semantics.

\section{Syntax}

Definition 1. (Epistemic Language) Let $\mathscr{A} t$ be a set of atomic formulas and $J$ a set of agents. The language we will be using is defined by:

$$
\varphi:=p|\neg \varphi| \varphi \wedge \varphi \mid K_{j} \varphi
$$

where $p \in \mathscr{A} t$ and $j \in J$.

We use the common definitions of $\vee, \rightarrow$, and $\leftrightarrow$ in terms of $\neg$ and $\wedge$. The intended reading of the epistemic operator $K_{j} \varphi$ is "Agent $j$ knows that $\varphi$ ". As a convention, an epistemic formula will be any 
formula containing at least one occurrence of an epistemic operator, and a non-epistemic formula any formula that contains none.

As should be obvious, the epistemic language that we will be using simply is that of standard epistemic logic.

Semantics Its semantics, however, will differ from the standard Kripke semantics in that it will include (i) a set $\mathscr{C}$ of contexts, and (ii) a function $\mathscr{R}$ of relevance determining the worlds that are relevant in each context:

Definition 2. (Contextual Model) A contextual model for the epistemic language is a structure $\mathscr{M}=$ $\left\langle W,\left\{\mathscr{K}_{j}: j \in J\right\}, \mathscr{C}, \mathscr{R}, V\right\rangle$ where (i) $W$ is a non-empty set of worlds, (ii) $\mathscr{K}_{j} \subseteq W \times W$ is a relation of epistemic accessibility (for each $j \in J$ ), (iii) $\mathscr{C}=\left\{c_{i}: i \in I\right\}$ is a non-empty set of contexts, which may be finite or not, such that $J \subseteq I$, (iv) $\mathscr{R}: \mathscr{C} \rightarrow \wp(W)^{W}$ is a function of contextual relevance that associates with each context $c_{i}$, for each world $w$, the set of worlds that are relevant in $c_{i}$ for $w$, and (v) $V: \mathscr{A} t \rightarrow \wp(W)$ is a valuation associating with each atom $p$ the set of worlds in which $p$ holds.

Remark. A context $c_{i}$ can be connected with an agent, i.e., when $i \in J$. But nothing prevents us from connecting contexts with groups of agents instead of individual agents, or with conversations, etc. Also, the semantics considered here remains neutral as to the nature of contexts. (A possible modelling inspired by Discourse Representation Theory and whereby contexts can evolve through time over the course of several assertions will be described in Section 4).

The idea, then, is to relativize truth not only to (a model and) a world as in standard Kripke semantics, but also to a context:

Definition 3. (Truth) Given a contextual model $\mathscr{M}=\left\langle W,\left\{\mathscr{K}_{j}: j \in J\right\}, \mathscr{C}, \mathscr{R}, V\right\rangle$, a state $w \in W$, and a formula $\varphi$ in the epistemic language, we can define $\mathscr{M}, c, w=\varphi$ in four possible ways, depending on the clause we choose for epistemic formulas, as follows:

$$
\begin{aligned}
& \mathscr{M}, c, w \models p \text { iff } w \in V(p) \\
& \mathscr{M}, c, w \models \neg \varphi \text { iff } \mathscr{M}, c, w \neq \varphi \\
& \mathscr{M}, c, w \models \varphi \wedge \psi \text { iff } \mathscr{M}, c, w \models \varphi \text { and } \mathscr{M}, c, w \models \psi \\
& \mathscr{M}, c_{i}, w \models K_{j} \varphi \text { iff for every } w^{\prime}, \text { if } \mathscr{K}_{j} w w^{\prime} \text { and } w^{\prime} \in \mathscr{R}\left(c_{k}\right)(w) \text { then } \mathscr{M}, c_{l}, w^{\prime} \models \varphi \text { with } \\
& \text { either one of the following options: } \\
& \qquad \begin{array}{l}
\text { 1.1. } \quad k=l=i \\
\text { 1.2. } \quad k=i, l=j \\
\text { 2.1. } \quad k=j, l=i \\
\text { 2.2. } \quad k=l=j
\end{array}
\end{aligned}
$$$$
\mathscr{M}, c, w \models \varphi \wedge \psi \text { iff } \mathscr{M}, c, w \models \varphi \text { and } \mathscr{M}, c, w \models \psi
$$
either one of the following options:

To refer specifically to one of the four resulting definitions, we will subscript $\models$ with the appropriate number: $\mathscr{M}, c, w \models_{1.2} \varphi, \mathscr{M}, c, w \models_{2.2} \varphi$, etc. We will sometimes group the notions two-by-two, letting $\models_{-.2}$ refer unspecifically to the $\models_{1.2}$ case or the $\models_{2.2}$ case, for instance; and $\models$ will refer indifferently to any one of the four notions. ${ }^{2}$

\footnotetext{
${ }^{2}$ We mention all four logically possible options here for the sake of exhaustivity, although we will not appeal to all of them. To be precise, option 2.1 will not be relevant to our purpose. See footnote 6 .
} 
The Properties of Knowledge in Contextual Models We may wonder if the following common axioms and inference rules are preserved in our contextual models, and if not, what condition(s) must be imposed on the relevance function in order to restore them?

$$
\begin{array}{ll}
\mathrm{K} & \models\left(K_{j} \varphi \wedge K_{j}(\varphi \rightarrow \psi)\right) \rightarrow K \psi \\
\mathrm{RN} & \text { If } \models \varphi \text { then }=K_{j} \varphi \\
\mathrm{T} & \models K_{j} \varphi \rightarrow \varphi \\
\mathrm{D} & \models K_{j} \varphi \rightarrow \neg K_{j} \neg \varphi, \text { or } \models \neg K_{j} \perp \\
4 & \models K_{j} \varphi \rightarrow K_{j} K_{j} \varphi \\
5 & \models \neg K_{j} \varphi \rightarrow K_{j} \neg K_{j} \varphi \\
\mathrm{B} & \models \varphi \rightarrow K_{j} \neg K_{j} \neg \varphi
\end{array}
$$

To answer this question, let us assume the following convention. In a given contextual model, from the relevance function $\mathscr{R}$ and a context $c_{k}$, a new accessibility relation $R_{c_{k}}$ can be defined by:

$$
\forall w . \forall w^{\prime} . \mathscr{R}_{c_{k}} w w^{\prime} \Leftrightarrow w^{\prime} \in \mathscr{R}\left(c_{k}\right)(w) .
$$

Then, clause (iv) for epistemic formulas can be rewritten by means of the intersection $\mathscr{K}_{j}^{k}=\mathscr{K}_{j} \cap \mathscr{R}_{c_{k}}$ of the two accessibility relations:

$$
\begin{aligned}
& \mathscr{M}, c_{i}, w \models K_{j} \varphi \text { iff for all } w^{\prime} \in W, \text { if } \mathscr{K}_{j}^{k} w w^{\prime} \text { then } \mathscr{M}, c_{l}, w^{\prime} \models \varphi \\
& \text { (with some conditions on } k \text { and } l \text { ) }
\end{aligned}
$$

Let $\mathfrak{M}_{n}$ be the class of all (unrestricted) contextual models for $n$ agents regardless of the specific choice among options (1.1) to (2.2). It is easy to see that the following proposition holds:

Proposition 4. Both the axiom $(K)$ and the necessitation rule $(\mathrm{RN})$ are valid with respect to $\mathfrak{M}_{h}$ :

$$
\begin{aligned}
& \mathfrak{M}_{n}=\left(K_{j} \varphi \wedge K_{j}(\varphi \rightarrow \psi)\right) \rightarrow K_{j} \psi \\
& \text { If } \mathfrak{M}_{n}=\varphi \text { then } \mathfrak{M}_{n}=K_{j} \varphi
\end{aligned}
$$

Things get more complex when we turn to the additional possible properties. We will give only sufficient conditions for preserving these properties in contextual models. Two cases are to be systematically distinguished:

1. A simple case has to do with the (-.1) definitions, which by (iv') amount to:

$$
\text { (iv'/-.1) } \quad \mathscr{M}, c_{i}, w \models_{-.1} K_{j} \varphi \text { iff for all } w^{\prime} \in W \text {, if } \mathscr{K}_{j}^{k} w w^{\prime} \text { then } \mathscr{M}, c_{i},\left.w^{\prime}\right|_{-.1} \varphi \text {. }
$$

Here, the only role of context $c_{i}$ is to set the value of $k$. The definition is strictly equivalent to that of truth in the standard Kripke model $\mathscr{M}=\left\langle W,\left\{\mathscr{K}_{j}: j \in J\right\}, V\right\rangle$ that corresponds with the contextual model $\mathscr{M}=\left\langle W,\left\{\mathscr{K}_{j}: j \in J\right\}, \mathscr{C}, \mathscr{R}, V\right\rangle$, for an operator $K_{j}^{k}$ with accessibility relation $\mathscr{K}_{j}^{k}$ :

$\mathscr{M}, c_{i}, w=_{-.1} K_{j} \varphi \Leftrightarrow \mathscr{M}^{\prime}, w=K_{j}^{k} \varphi$.

Intuitively, contextual models will preserve for $K_{j}$, grosso modo, the principles corresponding to the properties of $\mathscr{K}_{j}$ that are preserved by $\mathscr{K}_{j}^{k}$. To be precise: 
Proposition 5. The axioms (T), (B), (4), and (5) are (1.1)- and (2.1)-valid in contextual models $\mathscr{M}$ whose relations $K_{j}$ and $\mathscr{R}_{c_{k}}$ are all respectively reflexive, symmetric, transitive, and Euclidian:

(T) $\left.\mathscr{M}_{-.1} K_{j} \varphi \rightarrow \varphi\right) \quad \Leftrightarrow \quad \mathscr{K}_{j}$ is reflexive and $w \in \mathscr{R}\left(c_{k}\right)(w)$ (for all $w$ )

(B) $\quad \mathscr{M} \vDash_{-.1} \varphi \rightarrow K_{j} \neg K_{j} \neg \varphi \quad \Leftrightarrow \quad \mathscr{K}_{j}$ is symmetric and $w \in \mathscr{R}\left(c_{k}\right)\left(w^{\prime}\right) \Rightarrow w^{\prime} \in \mathscr{R}\left(c_{k}\right)(w)$

(4) $\mathscr{M} \vDash_{-.1} K_{j} \varphi \rightarrow K_{j} K_{j} \varphi \quad \Leftrightarrow \quad \mathscr{K}_{j}$ is transitive and $w \in \mathscr{R}\left(c_{k}\right)\left(w^{\prime}\right) \& w^{\prime} \in \mathscr{R}\left(c_{k}\right)\left(w^{\prime \prime}\right)$ $\Rightarrow w \in \mathscr{R}\left(c_{k}\right)\left(w^{\prime \prime}\right)$

(5) $\quad \mathscr{M} \vDash_{-.1} \neg K_{j} \varphi \rightarrow K_{j} \neg K_{j} \varphi \quad \Leftrightarrow \quad \mathscr{K}_{j}$ is Euclidian and $w \in \mathscr{R}\left(c_{k}\right)\left(w^{\prime}\right) \& w \in \mathscr{R}\left(c_{k}\right)\left(w^{\prime \prime}\right)$ $\Rightarrow w^{\prime} \in \mathscr{R}\left(c_{k}\right)\left(w^{\prime \prime}\right)$

Making $K_{j}$ and $\mathscr{R}_{c_{k}}$ serial is not sufficient for ensuring the validity of $(D)$.

2. The case is more complicated with the (-.2) definitions, which by (iv') again amount to:

(iv'/-.2) $\quad \mathscr{M}, c_{i}, w \vDash_{-.2} K_{j} \varphi$ iff for all $w^{\prime} \in W$, if $\mathscr{K}_{j}^{k} w w^{\prime}$ then $\mathscr{M}, c_{j}, w^{\prime} \vDash_{-.2} \varphi$.

The difficulty here has to do with formulas with embedded modalities, since their truth will depend on several contexts. We illustrate this with axioms (T), (4), and (5):

Proposition 6. Schema (T) is neither (1.2)-valid nor (2.2)-valid in contextual models $\mathscr{M}$ with reflexive $K_{j}$ and $\mathscr{R}_{c_{k}}$ relations. Nevertheless, the following instances of $(T)$ hold in these models:

- $\mathscr{M}, c_{i}, w \vDash_{-.2} K_{j} \phi \rightarrow \phi$, for $\phi$ a non-epistemic formula;

- $\mathscr{M}, c_{i}, w \vDash_{-.2} K_{j} \phi \rightarrow \phi$ if $\mathscr{R}_{c_{i}} \subseteq \mathscr{R}_{c_{j}}$, for $\phi$ an epistemic formula in disjunctive normal form with no negated epistemic operator in it;

- $\mathscr{M}, c_{i}, w \vDash_{-.2} K_{j} \phi \rightarrow \phi$ if $\mathscr{R}_{c_{i}}=\mathscr{R}_{c_{j}}$, for $\phi$ an epistemic formula in normal disjunctive form with at least one negated epistemic operator.

Proposition 7. Validity of (4):

- Schema (4) is (2.2)-valid in contextual models $\mathscr{M}$ with reflexive $K_{j}$ and $\mathscr{R}_{c_{k}}$ relations;

- Schema (4) is not (1.2)-valid in contextual models $\mathscr{M}$ with transitive $K_{j}$ and $\mathscr{R}_{c_{k}}$ relations. Nonetheless, the following instantiation of (4) holds in such models: $\mathscr{M}, q, w \vDash_{1.2} K_{j} \phi \rightarrow$ $K_{j} K_{j} \phi$ if $\mathscr{R}_{c_{j}} \subseteq \mathscr{R}_{c_{i}}$.

Proposition 8. Validity of (5):

- Schema (5) is (2.2)-valid in contextual models $\mathscr{M}$ with Euclidian $K_{j}$ and $\mathscr{R}_{c_{k}}$ relations;

- Schema (5) is not (1.2)-valid in contextual models $\mathscr{M}$ with Euclidian $K_{j}$ and $\mathscr{R}_{c_{k}}$ relations. However, in such models the following instantiation of (5) holds: $\mathscr{M}, c, w \vDash_{1.2} \neg K_{j} \phi \rightarrow$ $K_{j} \neg K_{j} \phi$ if $\mathscr{R}_{c_{j}} \subseteq \mathscr{R}_{c_{i}}$.

We add a final technical remark:

Proposition 9. (Reduction to Standard Kripke Semantics) Definition (1.1) with w-constant relevance function $\mathscr{R}$ - i.e. such that for any context $c, \mathscr{R}(c)$ is constant - reduces to a case of standard Kripkean semantics.

The proofs of the above propositions are in the Appendix.

\subsection{The Formal Framework Applied}

We now give an epistemological interpretation of our formalism and an application of it in capturing, in a unique frame of comparison, the various epistemological positions described in Section2. 


\subsubsection{Elements of Epistemological Interpretation}

Interpreting the $\mathscr{K}_{j}$-s We propose to interpret the accessibility relations $\mathscr{K}_{j}$ in terms of epistemic indiscernibility, that is, we have $\mathscr{K}_{j} w w^{\prime}$ iff agent $j$ cannot tell $w$ from $w^{\prime}$ on the (sole) basis of what he knows. If $\varphi$ holds in a $\mathscr{K}_{j}$-accessible world thus interpreted, then for all $j$ knows, it might be that $\varphi$; in other words, it is epistemically possible that $\varphi$.

A question that naturally arises is what kind of relation is the epistemic accessibility relation. Here, our answer is that the $\mathscr{K}_{j}$ are to be construed as equivalence relations - i.e. reflexive, symmetric, and transitive -, thus following the common tendency in the logico-epistemic literature? The main epistemological reason for this is that as a general rule, epistemologists grant to the skeptic the premiss that their skeptical worlds are epistemically indiscernible from the actual world, that is, are exactly the same as the actual world with respect to whatever evidence or information we may have; and being exactly the same as is an equivalence relation.

Interpreting $\mathscr{R} \quad$ The relevance function $\mathscr{R}$ allows us to capture the idea of epistemic standards and their contextual variability. Indeed, it makes it possible to represent, for a given situation or world, the set of possible worlds that are relevant relative to a context, where the appropriate context (reference context, agent's context, etc.) depends on which definition we select for $=$. Given a world $w$, two contexts $c, c^{\prime}$ can be associated with two sets of contextually relevant worlds, $\mathscr{R}(c)(w)$ and $\mathscr{R}(c)(w)$. When $\mathscr{R}(c)(w) \subseteq \mathscr{R}\left(c^{\prime}\right)(w)$, the truth of an epistemic formula relative to $c^{\prime}$ will be more difficult to obtain than relative to $c$. So, each context can be understood as determining via $\mathscr{R}$ a certain level of epistemic requirement. The strengthening - resp. the weakening - of epistemic standards will thus translate, in our framework, as an extension - resp. a restriction - of the set of relevant worlds.

Epistemic Accessibility and Contextual Relevance The set of contextually relevant worlds for an agent $j$ in a world $w$ cannot be strictly included in the set of epistemically possible worlds for $j$. For $j$ could know that $\varphi$, hence having his accessibility relation $\mathscr{K}_{j}$ restricted to $\varphi$-worlds, yet also know that another agent $k$ does not know that $\varphi$ when some $\neg \varphi$-world is accessible by $\mathscr{T}$; this requires that $j$ be able to consider some of these $\neg \varphi$-worlds although they are not accessible to him. So, letting $\mathscr{K}(j)(w)=\left\{w^{\prime}: w^{\prime} \in W \& \mathscr{K}_{j} w w^{\prime}\right\}$ be the set of worlds that are epistemically accessible to agent $j$, what we must not have is this: $\mathscr{R}\left(c_{j}\right)(w) \subsetneq \mathscr{K}(j)(w)$.

Interpreting the $c_{i}$-s Crucial to the epistemological use we want to make of our formal framework is the distinction between the subject and the attributor of knowledge. In both cases, however, we are dealing with an agent. In one case, it is the agent $i$ whom knowledge of a proposition $\varphi$ is being attributed to; in the other case, it is the agent $j$ who attributes knowledge of $\varphi$ to $i$. An agent can also attribute knowledge of a proposition to himself in the first person, as in "I know this-or-that". In this case, he is both knowledge attributor and knowing subject.

To account for these various cases in our framework, we adopt the following conventions. In an evaluation of the form $\mathscr{M}, c_{i}, w \models K_{j} \varphi$, (i) at the most general level, context $c_{i}$ will be called the "context of reference", and $c_{j}$ the "context of agent $j$ "; (ii) for $i \in I$, on the one hand, agent $i$ will be associated with the "attributor" and $c_{i}$ referred to as "attributor $i$ 's context", and on the other hand, agent $j$ will be associated with the "subject" and $c_{j}$ referred to as "subject $j$ 's context"; and (iii) when considering $\mathscr{R}\left(c_{k}\right)(w)$, we shall refer to $c_{k}$ as the "attributor's context" or as the "subject's context" depending on whether $k=i$ or $k=j$.

\footnotetext{
${ }^{3}$ See for instance the reference handbook [12] on epistemic logic by Fagin et al. Dissenting views, however, exist, as expressed by Hintikka in [22], and more recently by Stalnaker in [34].
} 


\subsubsection{Epistemological Interpretation of the $=-s$}

We can now consider the various definitions of $\models$ and connect them with the various epistemological positions mentioned earlier. As we will see, one of the major advantages of our formalism is that it show what answer each of these positions can give to a problem often underestimated in the epistemological literature, viz. that of embedded epistemic operators.

A first observation is that there seems to be no noticeable difference between definition (1.1) and definition (1.2) as far as their epistemological interpretation is concerned. The former:

$$
\mathscr{M}, c_{i}, w \models_{1.1} K_{j} \varphi \text { iff for every } w^{\prime} \text {, if } \mathscr{K}_{j} w w^{\prime} \text { and } w^{\prime} \in \mathscr{R}\left(c_{i}\right)(w) \text { then } \mathscr{M}, c_{i}, w^{\prime} \models \varphi
$$

says, basically, that I (= attributor) can truly say that you (= subject) know that $\varphi$ when $I$ can truly say, against my epistemic standards, that given your evidence, you know that $\varphi$; and the latter definition:

$$
\mathscr{M}, c_{i}, w=_{1.2} K_{j} \varphi \text { iff for every } w^{\prime} \text {, if } \mathscr{K}_{j} w w^{\prime} \text { and } w^{\prime} \in \mathscr{R}\left(c_{i}\right)(w) \text { then } \mathscr{M}, c_{j}, w^{\prime}=\varphi
$$

says, basically, that I (= attributor) can truly say that you (= subject) know that $\varphi$ when you can truly say, against $m y$ epistemic standards, that given your evidence, you know that $\varphi$. Now, on both definitions, whether a world is relevant or not depends on the attributor's context.

However, an important difference shows between the two definitions when we turn to formulas with embedded occurrences of epistemic operators, e.g. formulas like $K_{1} K_{2} \ldots K_{m} \varphi$.

Definition (1.1) as Invariantism Regarding embedded epistemic operators, definition (1.1) yields:

$$
\begin{gathered}
\mathscr{M}, c_{1}, w \models_{1.1} K_{2} K_{3} \varphi \text { iff for every } w^{\prime}, w^{\prime \prime}, \text { if } \mathscr{K}_{2} w w^{\prime}, \mathscr{K}_{3} w^{\prime} w^{\prime \prime}, w^{\prime} \in \mathscr{R}\left(c_{1}\right)(w) \text { and } w^{\prime \prime} \in \mathscr{R}\left(c_{1}\right)\left(w^{\prime}\right), \\
\text { then } \mathscr{M}, c_{1}, w^{\prime \prime} \models_{1.1} \varphi .
\end{gathered}
$$

which amounts to saying this: When attributor 1 says that subject 2 knows that subject 3 knows that $\varphi$, for 1's attribution to be true, it is always exactly the same standards as 2 that 3 must satisfy, that is to say, those in place in attributor 1's context. We propose to associate this definition with the two (insensitive) invariantist positions distinguished in Section2, viz. skepticism and anti-skepticism. Both hold that the standards for making a true knowledge attribution are the same always and everywhere, regardless of who is attributing and who is being attributed knowledge. Simply, the former holds that those standards are too demanding for any such attribution to ever come out true, while the latter says they are lax enough to make (most of our) everyday knowledge attributions true.

The difference can be expressed formally in our framework by putting different constraints on the relevance function $\mathscr{R}$ :

- For skepticism, the constraint that $\mathscr{R}\left(c_{i}\right)(w)=W$, for any $i$ and any $w$. This means that whatever the attributor's context, the corresponding relevance set is always the entire set of all logically possible worlds, including, of course, such far-fetched worlds as those described by the Evil Genius or the Brain in a Vat hypotheses, that cannot be eliminated on the basis of our limited epistemic capabilities. In assuming this constraint, the skeptic pretends to be a god, and that people can reason on other people's knowledge only if they are gods themselves. Skeptical epistemic logic is epistemic logic for divine agents.

- For anti-skepticism, the constraint that for any $i$ and any $w, \mathscr{R}\left(c_{i}\right)(w)=W *$, for some proper subset $W *$ of $W$, seems to be a minimum requirement, which nonetheless makes it less impossible for non-divine epistemic agents like us to truly claim to knowledge. At least in some cases, all contextually relevant possibilities of error can be excluded. A further requirement - given here informally as a first approximation - will have to be that $W *$ be a set of epistemically accessible worlds where most propositions we ordinarily think we have knowledge of are true (e.g. that we have hands). 
As should be clear, either one of these constraints will ensure that the set of epistemically relevant worlds is constant across contexts, that is, for any $w, i$, and $j, R\left(c_{i}\right)(w)=R\left(c_{j}\right)(w)$, which justifies applying to them the label "invariantism". ${ }^{4}$

Definition (1.2) as Contextualism In contrast with definition (1.1), with embedded epistemic operators, definition (1.2) yields:

$$
\begin{gathered}
\mathscr{M}, c_{1}, w=_{1.2} K_{2} K_{3} \varphi \text { iff for every } w^{\prime}, w^{\prime \prime}, \text { if } \mathscr{K}_{2} w w^{\prime}, \mathscr{K}_{3} w^{\prime} w^{\prime \prime}, w^{\prime} \in \mathscr{R}\left(c_{1}\right)(w) \text { and } w^{\prime \prime} \in \mathscr{R}\left(c_{2}\right)\left(w^{\prime}\right), \\
\text { then } \mathscr{M}, c_{3}, w^{\prime \prime} \models_{1.2} \varphi .
\end{gathered}
$$

This entails that if an attributor 1 says that subject 2 knows that subject 3 knows that $\varphi$, for 1 's attribution to be true, the standards that 2 must satisfy for 1's attribution to be true will be those in place in 1's context, while those that 3 must satisfy for it to be true that he knows $\varphi$ will be those in place in subject 2's context, not attributor 1's context; and the two sets of standards might well be different in their requirements. This, on our opinion, is what we may and must expect from the behavior of genuine contextualist agents (who assume themselves as such): a contextualist agent ought to be aware not only reasons about other agents' knowledge in the light of his own standards, but also that the other agents do and ought to do the same too. We therefore suggest associating definition (1.2) with (genuine, selfassumed, coherent) contextualism. 5 To capture formally the contextualist idea that the views of the skeptic and the anti-skeptic are not incompatible, we can simply put on $\mathscr{R}$ the softer constraint that $\mathscr{R}\left(c_{i}\right)(w) \subseteq W$, so that when $c_{i}$ is a philosophical context, $\mathscr{R}$ yields the set of all logicaly possible worlds, and when it is an everyday context, $\mathscr{R}$ yields a proper subset of those worlds - preferentially with epistemically possible worlds where most of what we ordinarily think we know is true.

Interestingly, so identifying contextualism with definition (1.2) brings to the fore that contextualism renders a relatively uncontroversial epistemic principle really problematic, viz. the "veridicality principle" whereby knowledge requires truth. As Proposition 6 points out, the formal version of this principle - schema (T) $K \varphi \rightarrow \varphi$-, is (1.2)-valid in contextual models with reflexive contextualized accessibility relations $\mathscr{K}_{i}^{k}$ only when $\varphi$ is non-epistemic. This restriction of the implication from knowledge to truth to non-epistemic "facts" is totally in line with the spirit of contextualism for which there are no such things as "epistemic facts". "Know" does not relate to things like knowledga, knowledge $2, \ldots$ that would exist objectively. Since one can count as knowing with respect to one attributor, yet as not knowing with respect to another, the contextualist's concept of knowledge cannot be descriptive, but purely evaluative. So, if knowledge implies truth, it can only be non-epistemic truth. This is a consequence of contextualism that our proposed formal framework makes clearly salient.

Definition (2.2) as Subjectivism According to Proposition 6, we get similar restricted veridicality with definition (2.2.):

$$
\mathscr{M}, c_{i}, w \models_{2.2} K_{j} \varphi \text { iff for every } w^{\prime} \text {, if } \mathscr{K}_{j} w w^{\prime} \text { and } w^{\prime} \in \mathscr{R}\left(c_{j}\right)(w) \text { then } \mathscr{M}, c_{j}, w^{\prime}=\varphi
$$

This definition, however, is very different from the contextualist spirit of definition (1.2), and closer on our opinion to the spirit of subjectivism. What it says is that only the subject, $j$, matters. When we attribute knowledge of a proposition to a subject, it is the standards in place in his context, not ours, that matters for the truth or falsity of our attribution. Now, his context is also that in which he himself settles

\footnotetext{
${ }^{4}$ Note in passing that they make the epistemic relevance set constant across worlds too, differing in this respect from "non-absolutist", "circumstance-sensitive" forms of invariantism à la Dretske or Nozick, not treated here.

${ }^{5}$ Here, some proponents of contextualism might disagree as they would be willing to maintain that contextualism is true despite most people lacking awareness of this fact, and being semantically blind to the context-dependence of knowledge ascriptions. This is how, for instance, DeRose seems to conceive of the position. In [28], we insist on the distinction between these two construals of the contextualist stance and explore their respective bearings on the issue of epistemic factivity.
} 
on the truth of the proposition whose knowledge we attribute to him. This means that on this definition (unlike contextualist definition (1.2)), there are epistemic facts: it is the subject's knowledge that varies from context to context, not merely the truth of our attributions of knowledge to him. Depending on what's at stake in his context, a subject can possess, lack, or lose possession of his knowledge. This is in line with the characterization we gave earlier of subjectivism.

Now, although (not) knowing something is an epistemic fact, someone $A$ 's knowing that someone else $B$ knows something $p$ does not entail that $B$ knows $p$. For suppose it is true that $A$ knows that $B$ knows $p$. Then, $A$ must somehow satisfy the standards in place in his own context for knowing that $B$ knows $p$. Does this mean that $B$ thereby knows $p$ ? No, for nothing in subjectivism prohibits a priori that the standards in place in $B$ 's context be no more demanding as those in place in $A$ 's context. So, if subjectivism is true, veridicality cannot extend to one's knowledge of someone else's knowledge. This is exactly what definition (2.2) says, and further motivation for associating subjectivism with it.

\section{Dynamic Formalism}

\subsection{The Main Idea}

The notion of context in the previous section was left totally unspecified: we simply took it as a point $\epsilon$ in a set $\mathscr{C}$. In this section we propose a dynamic formalization of the notion, inspired by the semantics of discourse for natural languages.

The semantics of discourse, in particular Discourse Representation Theory (DRT), was invented by Kamp [23] to account for semantic phenomena specifically connected with discourse dynamics (as opposed to sentence dynamics) and not explainable in standard, say Montagovian, analysis: anaphora resolution, donkey sentences, etc. 'The "dynamic turn" in formal semantics has led to the creation of other formalisms too, like DPL (Dynamic Predicate Logic; see [16]) where the semantic value of an utterance is treated as a program modifying a context.

DRT builds an intermediate representational level between language and model, made of discourse representation structures (DRSs). Each DRS constitutes both (i) the context of interpretation and (ii) the update by this interpretation - yielding a new DRS. What matters here is the idea of introducing a representational level produced by the interpretation, which contains "syntactic traces" of the various assertions made in the course of a discourse.

\subsection{Pseudo-DRT for a Propositional Language}

For our purpose we will not need such a complex formalism as in DRT. We will not need a universe (due to the lack of individual variables) - except if we want to specify some features of extralinguistic context like the speaker, the place, etc., and in what follows we will simply dispense with such features. However, we will need and make use of a set of conditions preceded with a label (in the spirit of [14]).

As usual, a DRS (i.e. a context) will be represented by a box. For instance, the DRS produced by the interpretation of the following discourse:

$\varphi_{1}$; if $\varphi_{1}$ then agent $i$ knows that $\varphi_{2}$; therefore $i$ knows that $\varphi_{2}$

${ }^{6}$ As mentioned in footnote 2 , for our purposes, we do not need definition (2.1):

$$
\mathscr{M}, c_{i}, w \mid{ }_{2.1} K_{i} \varphi \text { iff for every } w^{\prime}, \text { if } \mathscr{K}_{j} w w^{\prime} \text { and } w^{\prime} \in \mathscr{R}\left(c_{j}\right)(w) \text { then } \mathscr{M}, c_{i}, w^{\prime}=\varphi
$$

which says that I (attributor) can truly say that you (=subject) know that $\varphi$ when $I$ can truly say, against your epistemic standards, that given your evidence, you know that $\varphi$. It might, however, reveal useful if we augmented the non-modal part of the language with indexical expressions in order to account for such knowledge ascriptions as "So-and-so knows that I am here" or "I know that you are there".

${ }^{7}$ For an overview, see [39]; for a more complete presentation, see [24]. 
will be represented by:

$$
c=\begin{array}{|l|l|}
\hline A & \varphi_{1}, \varphi_{1} \rightarrow K_{i} \varphi_{2}, K_{i} \varphi_{2} \\
\hline
\end{array}
$$

where the label $A$ is meant to indicate that the relevant context was produced by means of a sequence of assertions. (We do not introduce sub-DRSs since in a propositional language the question of accessibility between universes does not arise).

This context will be interpreted against a "proto-context", that is to say, a set of presuppositions consisting exclusively in literals, i.e. atoms or negation of atoms (of the form "Agent $i$ is not a brain in a vat", or "Agent $j$ 's vision is reliable"). This proto-context will be represented by a box labelled with $P$ :

$$
c^{\star}=\begin{array}{|l|l|}
\hline P & h_{1}, h_{2}, \ldots, h_{n} \\
\hline
\end{array}
$$

Several definitions are in order here.

Definition 10. A discourse in language $\mathscr{L}$ is a finite (ordered) sequence of formulas of $\mathscr{L}$ :

$$
D=\left\langle\phi_{1}, \ldots, \phi_{p}\right\rangle
$$

Definition 11. A (discourse) context for $\mathscr{L}$ is a pair consisting in a label $X$ and a set $\Sigma$ of formulas of $\mathscr{L}: c=\langle X, \Sigma\rangle$. Notation: \begin{tabular}{|l|l|}
\hline & $\Sigma$ \\
\hline
\end{tabular}

- An assertion context is a context labelled with $A:$\begin{tabular}{|l|l|}
\hline$A$ & $\varphi_{1}, \ldots, \varphi_{m}$ \\
\hline
\end{tabular}

- A proto-context, or presupposition context, is a context labelled with $P:$\begin{tabular}{|l|l|}
\hline$P$ & $h_{1}, h_{2}, \ldots, h_{n}$ \\
\hline
\end{tabular} , each of the formulas $h_{i}$ being a literal.

To refer to the labels, formulas, and atoms involved in a context, we use the following conventions: Notation 12. Writing conventions:

- The label of a context $c$ is written $L a b(c)$ - i.e. $\left.L a b \begin{array}{|l|l|}X & \Sigma\end{array}\right)=X$;

- The set of formulas of a context $c$ is written $F o(c)$ - i.e. $\left.F o \begin{array}{|l|l|}\hline X & \Sigma \\ \hline\end{array}\right)=\Sigma$;

- The set of atoms making up the formulas of a context $c$ is written $\operatorname{At}(c): \operatorname{At}(c) \in \wp(\mathscr{A} t)$.

Definition 13. The agglomeration of a formula $\phi$ with a context $c$, written $c+\phi$, is a binary function on $\mathscr{C} \times \mathscr{L}$ taking its values in $\mathscr{C}$ and defined as follows:

$$
\begin{array}{|l|l|l|}
\hline X & \varphi_{1}, \ldots, \varphi_{l} \\
\hline
\end{array}+\psi=\begin{array}{|l|l|}
\hline X & \varphi_{1}, \ldots, \varphi_{l}, \psi \\
\hline
\end{array}
$$

The foregoing definition entails that if a formula is already in a context, its agglomeration does not modify this context.

Definition 14. The representation of a discourse $D=\left\langle\phi_{1}, \ldots, \phi_{p}\right\rangle$ relative to a context $c, R(c, D)$, is a finite sequence of assertion contexts $\left\langle c_{0}, c_{1}, \ldots, c_{p}\right\rangle$ formed by successive agglomeration of the formulas of $D$, i.e. such that:

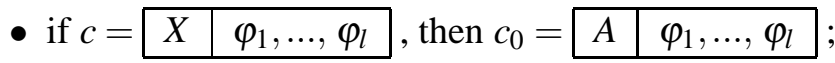

- for each index $i \in\{0, \ldots, p-1\}$, we have: $c_{i+1}=c_{i}+\varphi_{i+1}$.

Now that we can represent discourses - by a set of markers (the formulas of the context) representing the various assertions of a discourse -, we must consider the semantic interpretation of contexts. 
Definition 15. The semantic value $\llbracket c \rrbracket$ of a context $c$ in a Kripke model $\mathscr{M}=\langle W, \mathscr{K}, V\rangle$ is the set of possible worlds compatible with the formulas of $c$ :

$$
\text { If } c=\begin{array}{|l|l|}
\hline X & \varphi_{1}, \ldots, \varphi_{l}
\end{array} \text {, then } \llbracket c \rrbracket=\left\{w: w \in W \& \mathscr{M}, w=\left(\varphi_{1} \wedge \ldots \wedge \varphi_{l}\right)\right\} \text {. }
$$

What remains to be done is to introduce a dynamic component at the level of proto-contexts. A protocontext must enable regimentation of the presuppositions of a discourse, that is, of those statements whose truth is not put into question and which are not even made explicit in the discourse context. The discourse itself can make a presupposition explicit, put it into question, and thereby modify the proto-context.

Definition 16. The fusion of two contexts is a partial binary function \# : $\mathscr{C} \times \mathscr{C} \rightarrow \mathscr{C}$ such that, for any pair $\left\langle c_{1}, c_{2}\right\rangle$ where $c_{1}=$\begin{tabular}{|l|l|l|l|}
\hline$P$ & $h_{1}, h_{2}, \ldots, h_{n}$ & is a proto-context and $c_{2}=A$ & $\varphi_{1}, \varphi_{2}, \ldots, \varphi_{m}$ \\
\hline
\end{tabular} assertion context:

$$
c_{1} \# c_{2}=\begin{array}{|l|l|l|l|}
\hline P & h_{1}, h_{2}, \ldots, h_{n} \\
\hline
\end{array} \# \begin{array}{|l|l|l|}
\hline A & \varphi_{1}, \varphi_{2}, \ldots, \varphi_{m} \\
\hline
\end{array}
$$

with:

- $F o\left(c_{1} \# c_{2}\right) \subseteq F o\left(c_{1}\right)$;

- $A t\left(c_{1}\right) \backslash A t\left(c_{1} \# c_{2}\right)=A t\left(c_{1}\right) \cap A t\left(c_{2}\right)$.

In other words, fusion removes from the proto-context all those literals that are atoms or negation of atoms included in the assertion context. Based on this definition, we can then consider a new sequence of contexts resulting from the analysis of a discourse, viz. the sequence of proto-contexts which parallels the representation of the discourse:

Definition 17. Given a proto-context $c^{\star}$ and a discourse $D=\left\langle\phi_{1}, \ldots, \phi_{p}\right\rangle$ interpreted relative to an initial context $c$ - with representation $R(c, D)=\left\langle c_{0}, c_{1}, \ldots, c_{p}\right\rangle$-, we build the history of proto-context $c^{\star}$, written $H\left(c^{\star}, c, D\right)$, consisting in a sequence of proto-contexts $\left\langle c_{0}^{\star}, c_{1}^{\star}, \ldots, c_{p}^{\star}\right\rangle$ such that:

- $c_{0}^{\star}=c^{\star} \# c ;$

- for every index $i \in\{0, \ldots, p-1\}$, we have: $c_{i+1}^{\star}=c_{i}^{\star} \# c_{i+1}$.

A history of proto-contexts thus explains the progressive modification of the set of presuppositions by removing the literals that are made explicit (or whose negation is made explicit) in the discourse.

We can associate with a history of proto-contexts a ( $w$-constant) relevance function such that:

$$
\forall w: \mathscr{R}\left(c_{i}^{\star}\right)(w)=\llbracket c_{i}^{\star} \rrbracket .
$$

This function allows the set of contextually relevant worlds to evolve over the course of the interpretation of a discourse.

\subsection{Application}

The static formalism described in Section 3 runs into a problem faced by all systems of normal modal logic and having to do with the logical omniscience that follows from accepting axiom $\mathrm{K}$ - the epistemic closure principle - and the necessitation rule.

The DRT-based semantics just described allows us to overcome these difficulties. The effects of the necessitation rule can indeed be by-passed if we suppose that each assertion modifies the evaluation context. That is to say, although we do have $K \varphi_{1}, K\left(\varphi_{1} \rightarrow \varphi_{2}\right), K \varphi_{2}$ relative to a constant context, this is no longer the case when the context evolves over a sequence of assertions. 
We propose to evaluate $K \varphi_{1}$ relative to an initial empty context, $c=|A| \square$; we then evaluate the next formula $K\left(\varphi_{1} \rightarrow \varphi_{2}\right)$ relative to the new context produced by the agglomeration of $K \varphi_{1}$, viz.:

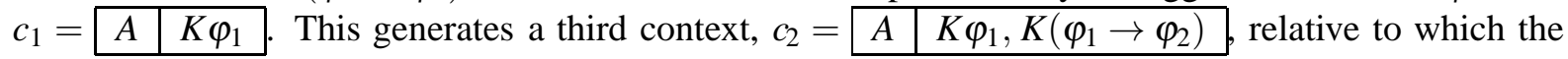
conclusion $K \varphi_{2}$ can be false, depending on the effect of the first two assertions on the history of the initial proto-context.

Example. To make things simple, let us assume an epistemic language with only one operator $K$ and a unique relation $\mathscr{K}$. Given a presupposition context containing $h_{1}=\neg r$, read as "The agent is not

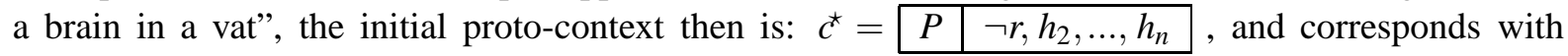
ordinary, rather lax epistemic standards, quite unlike those of the skeptic. Let us analyze the following (well-known) piece of discourse:

- $\varphi_{1}$. The agent knows that he has two hands.

- $\varphi_{2}$. If the agent knows that he has two hands then he knows that he is not a brain in a vat.

- $\varphi_{3}$. Therefore, the agent knows that he is not a brain in a vat.

Letting $p$ stand for the atom expressing that the agent has two hands, we get the following discourse: $D=\langle K p, K(p \rightarrow \neg r), K \neg r\rangle$. To interpret it, we suppose that neither $p$ nor $\neg p$ is part of the initial presuppositions $\left(p \notin A t\left(c^{\star}\right)\right)$. In the following figure the two sequences generated by $D$ are in two parallel columns:

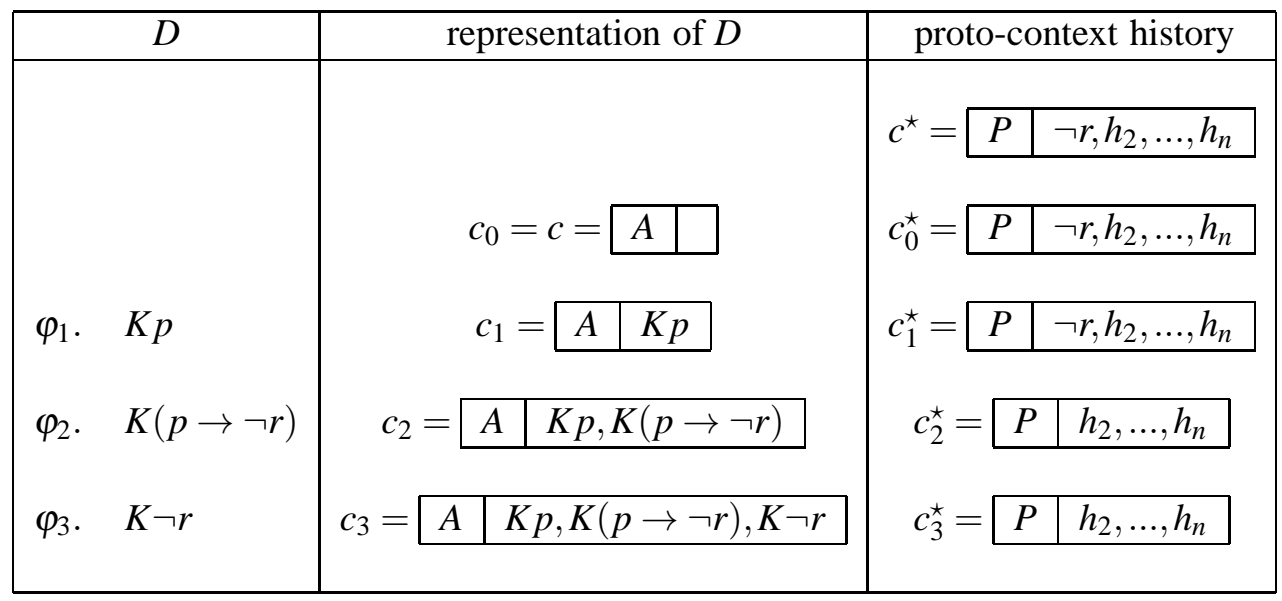

As mentioned above, we can put the history of the proto-context to use to define a relevance function, thereby obtaining a constant function on $c^{\star}, c_{0}^{\star}$, and $c_{1}^{\star}$, whose co-domain (the relevant worlds) extends from $c_{2}^{\star}$ :

$$
\mathscr{R}\left(c^{\star}\right)=\mathscr{R}\left(c_{0}^{\star}\right)=\mathscr{R}\left(c_{1}^{\star}\right)=\llbracket c^{\star} \rrbracket \subsetneq \llbracket c_{2}^{\star} \rrbracket=\mathscr{R}\left(c_{2}^{\star}\right)=\mathscr{R}\left(c_{3}^{\star}\right) .
$$

Each formula $\varphi_{i}$ of $D$ is interpreted against the previous context of the representation, $q_{-1}$. Let us suppose that the interpretation of the first two assertions is true in a given world $w$. Then:

$$
\begin{array}{ccl}
\mathscr{M}, c_{0}, w \models K p & \text { i.e.: } & \forall w^{\prime} \in \mathscr{R}\left(c_{0}^{\star}\right)(w)\left(=\llbracket c^{\star} \rrbracket\right): \mathscr{K} w, w^{\prime} \Rightarrow \mathscr{M}, c_{0}, w^{\prime} \models p \\
\mathscr{M}, c_{1}, w \models K(p \rightarrow \neg r) & \text { i.e: } & \forall w^{\prime} \in \mathscr{R}\left(c_{1}^{\star}\right)(w)\left(=\llbracket c^{\star} \rrbracket\right): \mathscr{K} w, w^{\prime} \Rightarrow \mathscr{M}, c_{1}, w^{\prime} \models(p \rightarrow \neg r)
\end{array}
$$

It follows that relative to $c_{1}$, the formula $K \neg r$ is true in $w$. But it is relative to $c_{2}$ that it is evaluated, whose class of relevant worlds is a proper extension of $\llbracket c^{\star} \rrbracket$ :

$$
\mathscr{M}, c_{2}, w=K \neg r \quad \Leftrightarrow \quad \forall w^{\prime \prime} \in \mathscr{R}\left(c_{2}^{\star}\right)(w)\left(=\llbracket c_{2}^{\star} \rrbracket\right): \mathscr{K} w, w^{\prime \prime} \Rightarrow \mathscr{M}, c_{2}, w^{\prime \prime} \models \neg r .
$$


So, for $K \neg \varphi$ to fail to hold in $w$, all we need is a world that is $\mathscr{K}$-accessible from $w$, where the agent is a brain in a vat, and that is relevant in context $c_{2}$. And in this context, unlike $c_{1}$, there can be one such world, as the following example shows.

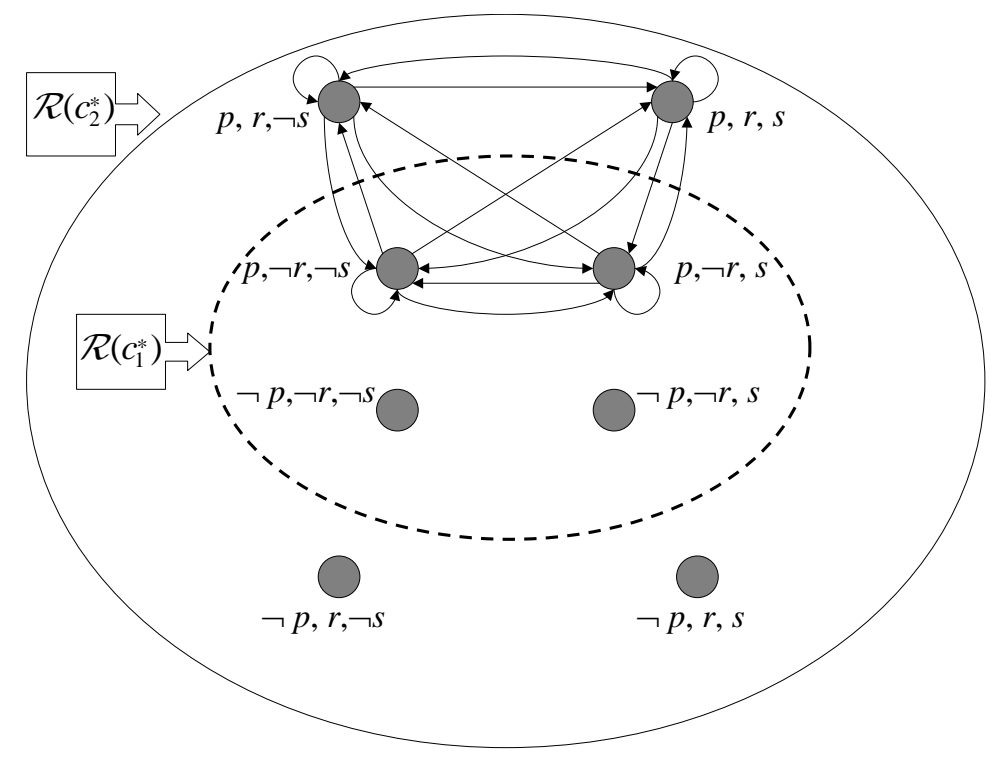

\subsection{Discourse Contexts and Belief Bases}

The dynamic approach just proposed is akin to the well-known AGM model of belief revision of B3] (for a recent overview, see [38]). We chose not to make use of the latter approach because it is not sensitive enough to the syntax of formulas, and is thereby too static, or so to speak.

Belief bases in the AGM model are deductively closed: this results in a level of idealization that is too high to deal with certain epistemological issues (see [17]), as we inherit ipso facto the problems of logical omniscience. By contrast, the DRT-inspired discourse contexts are finite - and even very limited - sets of formulas: only those formulas that directly represent assertions are introduced in an assertion context. A formula being in a context therefore does not imply that, say, all disjunctions containing that formula be in that context. Unlike belief bases, discourse contexts are not deductively closed.

The set of formulas of a given context $c, F o(c)$, nonetheless coincides with its deductive closure $(\mathrm{Cn}(\mathrm{c}))$ when it comes to evaluation. We can then consider describing agglomeration as an expansion, and fusion of a proto-context with an assertion context as a contraction of the proto-context.

Belief revision theories could also be of relevance at the level of assertion contexts when a discourse generates an inconsistency that calls for a revision of the DRS. Several connections can therefore be drawn between the approach developped in the present paper and the dominant approaches to doxastic dynamics in Artificial Intelligence.

\section{Conclusion}

In this paper we have laid out the foundations of a formal framework that uses the tools of epistemic logic to advance epistemological analysis.

Epistemologically speaking, the application of our framework to capturing various philosophical positions about knowledge will have to be extended so as to account for "assessment-sensitive" positions à la Macfarlane [29]- for whom epistemic standards vary with the context of the person who assesses 
for truth or falsity a knowledge ascription made by an attributor about a subject -, as well as such "circumstance-sensitive" positions à la Dretske or Nozick - for whom epistemic standards vary not with any context whatsoever, but with the world with respect to which the subject's epistemic position is being evaluated. This is left for future work.

Logically speaking, this time, the very framework of contextual models requires further exploration. We have here sketched a "deviant" two-dimensional semantics that augments the usual possible world structure with a relevance function. A step further would be to develop a syntax matching those models by introducing context operators of the form $\left[c_{i}\right]$ and $\left\langle c_{i}\right\rangle$ that would make it possible to refer explicitly to context-dependence directly in the object-language. ${ }^{8}$ Combining such operators with contextualized epistemic operators would allow us to capture various epistemological positions within the same contextual model, and therefore to account for the logical behavior of, say, a contextualist agent reasoning about the knowledge of a skeptical agent reasoning about an anti-skeptical agent. The contextual model framework could also be extended along further lines, e.g.:

- by exploring different axiom systems (S4, T, etc.) for defining the epistemic operators $K_{i}$;

- by adding an awareness operator, or a notion of similarity between worlds, or any other modification that might block epistemic closure already at the "static" level of the framework (as required by positions like Dretske's or Nozick's);

- by adding operators for belief and justification (possibly in the line of 2]);

- by adding appropriate alethic modalities to allow for the treatment of counterfactual epistemic statements like "Had Mary seen Paul at the party, she'd know it";

- by adding indexical symbols to the non-modal fragment of the language so as to account for statements like "Mary knows I'm in Paris" or "I know you're there".

This too is left for future work.

Generally speaking, studying how the epistemic language can be modified and enriched should allow us to provide a finer-grained modelling of the various positions that can be found in the epistemological literature within our framework of contextual models.

\section{References}

[1] Alchourrón C. E., Gardenfors P. \& D. Makinson, 1985 : 'On the Logic of Theory Change : Partial Meet Contraction and Revision Functions', Journal of Symbolic Logic 50 : 510-530.

[2] Artemov, S. \& E. Nogina, 2005 : 'Introducing Justification into Epistemic Logic', Journal of Logic and Computation 15 : 1059-1073.

[3] Austin, John, 1946 : 'Other Minds', Proceedings of the Aristotelian Society, Supplementary Volume $20: 148-187$.

[4] Cohen, S., 1999 : 'Contextualism, Skepticism, and the Structure of Reasons', Philosophical Perspectives $13: 57-89$.

[5] Cohen, S., 2000 : 'Contextualism and Skepticism', in E. Sosa \& E. Villanueva (Eds.), Scepticism : Philosophical issues Vol. 10, Basil Blackwell, Boston, 94-107. 34

[6] Crocco, G. \& A. Herzig, 2005 : 'Les opérations de changement basées sur le test de Ramsey’ in P. Livet (Ed.), Révision des croyances (Traité des sciences cognitives), Paris, Hermès.

\footnotetext{
${ }^{8}$ Work in this direction can be found in our paper on "contextual epistemic logic" [32].
} 
[7] DeRose, K., 1992 : 'Contextualism and Knowledge Attributions', Philosophy and Phenomenological Research 52 : 913-929.

[8] DeRose, K., 1995 : 'Solving the Skeptical Problem', The Philosophical Review 104 : 1-52.

[9] DeRose, K., 1999 : 'Contextualism : An Explanation and Defense', in J. Greco \& E. Sosa (Eds.), The Blackwell Guide to Epistemology, Blackwell Publishers, Malden, 187-205.

[10] DeRose, K., 2004 : 'Single Scoreboard Semantics’, Philosophical Studies 119, 1-21.

[11] Descartes, R., 1641 : Meditationes de prima philosophia, French translation by M. Beyssade as Méditations métaphysiques, 1990, Le livre de Poche, Paris.

[12] Fagin, R., Halpern, J. et al., 1995 : Reasoning about Knowledge, MIT Press, Cambridge.

[13] Frege, G., 1918 : 'Der Gedanke', French translation as "La pensée” by C. Imbert in G. Frege, Ecrits logiques et philosophiques, 1971, Seuil, Paris.

[14] Geurts B. \& E. Maier, 2003 : Layered DRT. ms, University of Nijmegen, November 7, 2003. Available at http ://www.ru.nl/filosofie/tfl/bart/papers/ldrt.pdf.

[15] Gottschling, V., 2004 : 'Keeping the Conversational Score : Constraints for an Optimal Contextualist Answer', Erkenntnis $61: 295-314$.

[16] Groenendijk, J. \& M. Stokhof, 1991 : 'Dynamic Predicate Logic', Linguistics and Philosophy 14 : 39-100.

[17] Hansson, S.O., 2003 : 'Ten philosophical problems in belief revision', Journal of Logic and Computation $13: 37-49$.

[18] Heller, M., 1999a : 'Relevant Alternatives and Closure', Australasian Journal of Philosophy 77, 196-208.

[19] Heller, M., 1999b : 'The Proper Role for Contextualism in an Anti-luck Epistemology', Philosophical Perspectives 13 : 115-29.

[20] Hendricks, V.F., 2004 : 'Hintikka on Epistemological Axiomatizations', in Daniel Kolak \& John Symons (Eds.) : Questions, Quantifiers and Quantum Physics : Essays on the Philosophy of Jaakko Hintikka, Springer, Dordrecht, 3-34.

[21] Hawthorne, J., 2004 : Knowledge and Lotteries, Oxford University Press, Oxford.

[22] Hintikka, J., 1962 : Knowledge and Belief. An Introduction to the Logic of the Two Notions, Cornell University Press, New York.

[23] Kamp, H., 1981: 'A Theory of Truth and Semantic Representation', in Stokhof, J. et al. (Eds.), Formal Methods in the Study of Language, Matematisch Centrum, Amsterdam, 277-322.

[24] Kamp, H. \& U. Reyle, 1993 : From Discourse To Logic, Kluwer, Dordrecht.

[25] Kaplan, D., 1989 : 'Demonstratives', in Almog, Perry, \& Wettstein, Themes from Kaplan, Oxford University Press, Oxford.

[26] Lewis, D., 1979 : 'Scorekeeping in a Language Game', Journal of Philosophical Logic 8 : 339-59.

[27] Lewis, D., 1996 : 'Elusive Knowledge', Australasian Journal of Philosophy 74 : 549- 567. 
[28] Lihoreau, F., and M. Rebuschi : 'Contextualism and the factivity of knowledge', in D. Lukasiewicz \& R. Pouivet (Eds.), Scientific Knowledge and Common Knowledge, Publishing House Epigram and University of Kazimierz Wielki Press, Bydgoszcz, 209-224.

[29] MacFarlane, J., 2005 : 'The Assessment Sensitivity of Knowledge Attributions', in Szabo \& Hawthorne (Eds.), Oxford Studies in Epistemology, Oxford University Press, Oxford.

[30] Montague, R., 1974 : Formal Philosophy : Selected Papers of Richard Montague, Yale University Press, New Haven.

[31] Putnam, H., 1981 : Reason, Truth, and History, Cambridge University Press, Cambridge.

[32] Rebuschi, M. \& F. Lihoreau, 2008 : 'Contextual Epistemic Logic'. In C. Degrémont, L. Keiff \& H. Rueckert (Eds.), Dialogues, Logics and Other Strange Things, College Publications.

[33] Schotch, P. K., 2000 : 'Skepticism and Epistemic Logic', Studia Logica 66 : 187-198.

[34] Stalnaker, R., 2006 : 'On Logics of Knowledge and Belief', Philosophical Studies 128 : 169-199.

[35] Stanley, J., 2005 : Knowledge and Practical Interests, Oxford University Press, Oxford.

[36] Unger, P., 1971 : ‘A Defense of Scepticism', The Philosophical Review 80 : 198-219.

[37] van Benthem, J., 2006 : 'Epistemic Logic and Epistemology : The State of Their Affairs', Philosophical Studies 128 : 49-76.

[38] van Ditmarsch, H., van der Hoek, W. \& B. Kooi, 2008 : Dynamic Epistemic Logic, Springer, Dordrecht.

[39] van Eijk, J., 2005. 'Discourse Representation Theory', Encyclopedia of Language and Linguistics (2nd Ed.), Elsevier.

\section{Appendix: Proofs}

Proposition 5 The axioms (T), (B), (4), and (5) are (1.1)- and (2.1)-valid in contextual models $\mathscr{M}$ whose relations $K_{j}$ and $\mathscr{R}_{c_{k}}$ are all respectively reflexive, symmetric, transitive, and Euclidian:

(T) $\left.\quad \mathscr{M} \vDash_{-.1} K_{j} \varphi \rightarrow \varphi\right) \quad \Leftrightarrow \quad \mathscr{K}_{j}$ is reflexive and $w \in \mathscr{R}\left(c_{k}\right)(w)$ (for all $\left.w\right)$

(B) $\quad \mathscr{M} \vDash_{-.1} \varphi \rightarrow K_{j} \neg K_{j} \neg \varphi \quad \Leftrightarrow \quad \mathscr{K}_{j}$ is symmetric and $w \in \mathscr{R}\left(c_{k}\right)\left(w^{\prime}\right) \Rightarrow w^{\prime} \in \mathscr{R}\left(c_{k}\right)(w)$

(4) $\mathscr{M} \vDash_{-.1} K_{j} \varphi \rightarrow K_{j} K_{j} \varphi \quad \Leftrightarrow \quad \mathscr{K}_{j}$ is transitive and $w \in \mathscr{R}\left(c_{k}\right)\left(w^{\prime}\right) \& w^{\prime} \in \mathscr{R}\left(c_{k}\right)\left(w^{\prime \prime}\right)$ $\Rightarrow w \in \mathscr{R}\left(c_{k}\right)\left(w^{\prime \prime}\right)$

(5) $\quad \mathscr{M} \vDash_{-.1} \neg K_{j} \varphi \rightarrow K_{j} \neg K_{j} \varphi \quad \Leftrightarrow \quad \mathscr{K}_{j}$ is Euclidian and $w \in \mathscr{R}\left(c_{k}\right)\left(w^{\prime}\right) \& w \in \mathscr{R}\left(c_{k}\right)\left(w^{\prime \prime}\right)$ $\Rightarrow w^{\prime} \in \mathscr{R}\left(c_{k}\right)\left(w^{\prime \prime}\right)$

Making $K_{j}$ and $\mathscr{R}_{c_{k}}$ serial is not sufficient for ensuring the validity of $(D)$.

Proof.

- Suppose that for any $j$ and $k, \mathscr{K}_{j}$ and $\mathscr{R}_{c_{k}}$ are reflexive. Since the intersection of two reflexive relations is itself reflexive, the intersection $\mathscr{K}_{j}^{k}$ of $\mathscr{K}_{j}$ and $\mathscr{R}_{c_{k}}$ must be reflexive. Therefore, $\mathscr{M}^{\prime}, w \vDash K_{j}^{k} \varphi \rightarrow \varphi$, which amounts to $\mathscr{M}, c_{i}, w \vDash_{-.1} K_{j} \varphi \rightarrow \varphi$.

- As to axioms (B), (4) and (5), we can likewise simply observe that symmetry, transitivity and euclidianity are preserved by the intersection of two relations.

- As to axiom (D), however, seriality is not so preserved. For instance, the two relations $S=\{\langle a, b\rangle,\langle b, b\rangle\}$ and $T=\{\langle a, b\rangle,\langle b, a\rangle\}$ are both serial, yet their intersection boils down to $\{\langle a, b\rangle\}$. 
Proposition 6 Schema $(T)$ is neither (1.2)-valid nor (2.2)-valid in contextual models $\mathscr{M}$ with reflexive $K_{j}$ and $\mathscr{R}_{c_{k}}$ relations. Nevertheless, the following instances of $(T)$ hold in these models:

- $\mathscr{M}, c_{i}, w \vDash_{-.2} K_{j} \phi \rightarrow \phi$, for $\phi$ a non-epistemic formula;

- $\mathscr{M}, c_{i}, w \vDash_{-.2} K_{j} \phi \rightarrow \phi$ if $\mathscr{R}_{c_{i}} \subseteq \mathscr{R}_{c_{j}}$, for $\phi$ an epistemic formula in disjunctive normal form with no negated epistemic operator in it;

- $\mathscr{M}, c_{i}, w \vDash_{-.2} K_{j} \phi \rightarrow \phi$ if $\mathscr{R}_{c_{i}}=\mathscr{R}_{c_{j}}$, for $\phi$ an epistemic formula in normal disjunctive form with at least one negated epistemic operator.

Proof.

- (T) is not (-.2)-valid: Let $\mathscr{M}$ be a contextual model with reflexive relations, $c$ a context, and $w$ a world s.t. for any given formula $\phi, \mathscr{M}, c_{i}, w \vDash_{-.2} K_{j} \phi$. Then, for every $w^{\prime}$ s.t. $\mathscr{K}_{j}^{k} w w^{\prime}, \mathscr{M}, c_{j}, w^{\prime} \vDash_{-.2} \phi$. Since $\mathscr{K}_{j}^{k}$ is reflexive, in particular $\mathscr{M}, c_{j}, w \vDash_{-.2} \phi$. Now, this does not imply that $\mathscr{M}, c_{i}, w \vDash_{-.2} \phi$ : for instance, if $\phi$ is of the form $K_{m} \psi$, there can be a world $w_{0}$ s.t. $\mathscr{R}_{c_{i}} w w_{0}, \neg \mathscr{R}_{c_{j}} w w_{0}$ where $\psi$ is not satisfied.

- Under the same assumptions, take $\phi$ to be non-epistemic. Then, from clauses (i)(iii) for $=$ in contextual models, the value of $\phi$ is independent from context. So, $\mathscr{M}, c_{j}, w \vDash_{-.2} \phi$ entails $\mathscr{M}, c_{i}, w \vDash_{-.2} \phi$. Therefore, (T)'s instantiation with non-epistemic formulas is (-.2)-valid.

- With the same assumptions again, let us further suppose (1) $\phi$ to be in normal disjunctive form with no negation of an epistemic operator, and (2) that $\mathscr{R}_{c_{i}} \subseteq \mathscr{R}_{c_{j}}$. Then, $\phi$ is a disjunction of formulas: $\phi_{h}=K_{h_{1}} \psi_{h_{1}} \wedge K_{h_{m}} \psi_{h_{m}} \wedge \psi_{h_{m+1}} \wedge \ldots \wedge \psi_{h_{m+n}}$, for $\psi_{h_{m+1}}, \ldots, \psi_{h_{m+n}}$ non-epistemic formulas. When $\mathscr{M}, c_{j}, w \vDash_{-.2} \phi$, we have two cases:

1. The truth of $\phi=\phi_{1} \vee \ldots \vee \phi_{k}$ rests on a sub-formula $\phi_{h}$ with no epistemic component. In this case, $\mathscr{M}, c_{j}, w \vDash_{-.2} \phi_{h}$ implies that $\mathscr{M}, c_{i}, w \vDash_{-.2} \phi_{h}$, hence that $\mathscr{M}, c_{i}, w \vDash_{-.2} \phi$.

2. The truth of $\phi=\phi_{1} \vee \ldots \vee \phi_{k}$ rests on a sub-formula $\phi_{h}$ with epistemic components, hence on the truth of the sub-formulas $K_{h_{1}} \psi_{h_{1}}, \ldots, K_{h_{m}} \psi_{h_{m}}$ of $\phi_{h}$. Therefore, for each index $e \in\left\{h_{1}, \ldots, h_{m}\right\}$, we have $\mathscr{M}, c_{j}, w \vDash_{-.2} K_{e} \psi_{e}$; in other words, for all $w^{\prime}$, if $\mathscr{K}_{e}^{k} w w^{\prime}$ then $\mathscr{M}, c_{e}, w^{\prime} \vDash_{-.2} \psi_{e}$. We (provisionally) distinguish the two (-.2) definitions:

2.2: $\quad \mathscr{M}, c_{j}, w \vDash_{2.2} K_{e} \psi_{e}$ equates: for all $w^{\prime}$, if $\mathscr{K}_{e}^{e} w w^{\prime}$ then $\mathscr{M}, c_{e}, w^{\prime} \vDash_{2.2}$ $\psi_{e}$; in other words, the truth condition of $K_{e} \psi_{e}$ is independent from context $c_{j}$; so, we also have: $\mathscr{M}, c_{i}, w \vDash_{2.2} K_{e} \psi_{e}$.

1.2: $\quad \mathscr{M}, c_{j}, w \vDash_{1.2} K_{e} \psi_{e}$ equates: for all $w^{\prime}$, if $\mathscr{K}_{e}^{j} w w^{\prime}$ then $\mathscr{M}, c_{e}, w^{\prime} \vDash_{1.2}$ $\psi_{e}$; here, the truth condition for $K_{e} \psi_{e}$ depends on context $c_{j}$. But we have assumed that $\mathscr{R}_{c_{i}} \subseteq \mathscr{R}_{c_{j}}$, which entails that $\mathscr{R}_{c_{i}} \cap \mathscr{K}_{e} \subseteq \mathscr{R}_{c_{j}} \cap$ $\mathscr{K}_{e}$, i.e. $\mathscr{K}_{e}^{i} \subseteq \mathscr{K}_{e}^{j}$. Therefore, for all $w^{\prime}$, if $\mathscr{K}_{e}^{i} w w^{\prime}$ then $\mathscr{K}_{e}^{j} w w^{\prime}$, hence $\mathscr{M}, c_{e}, w^{\prime} \vDash_{1.2} \psi_{e}$. This amounts to $\mathscr{M}, c_{i}, w \vDash_{1.2} K_{e} \psi_{e}$.

We have thus shown that for each of the epistemic components $K_{e} \psi_{e}$ of $\phi_{h}, \mathscr{M}, c_{i}, w \vDash_{-.2} K_{e} \psi_{e}$; for the non-epistemic components $\psi_{h_{m+1}}, \ldots, \psi_{h_{m+n}}$, truth is independent from context and transposes from $c_{j}$ to $c_{j}$. Hence, we have established that $\phi_{h}, \mathscr{M}, c_{i}, w \vDash_{-.2} \phi_{h}$, and therefore that $\mathscr{M}, c_{i}, w \vDash_{-.2} \phi$. Conclusion: we have $\mathscr{M}, c_{i}, w \vDash_{-.2} K_{j} \phi \rightarrow \phi$.

- The case of formulas $\phi=\phi_{1} \vee \ldots \vee \phi_{k}$ whose truth rests on conjunctive components $\phi_{t}$ including sub-formulas of the type $\neg K_{e} \psi_{e}$ require more than the mere inclusion of the sets of contextually relevant worlds. Assuming the identity of these sets guarantees the transition of truth from context to context. The proof is similar to the previous one. 
Proposition 7 Validity of (4):

- Schema (4) is (2.2)-valid in contextual models $\mathscr{M}$ with reflexive $K_{j}$ and $\mathscr{R}_{c_{k}}$ relations;

- Schema (4) is not (1.2)-valid in contextual models $\mathscr{M}$ with transitive $K_{j}$ and $\mathscr{R}_{c_{k}}$ relations. Nonetheless, the following instantiation of (4) holds in such models: $\mathscr{M}, q, w \vDash_{1.2} K_{j} \phi \rightarrow K_{j} K_{j} \phi$ if $\mathscr{R}_{c_{j}} \subseteq \mathscr{R}_{c_{i}}$.

Proof.

(2.2): $\quad$ Let $\mathscr{M}$ be a contextual model with transitive relations, $c_{i}$ a context, $w$ a world, and $\phi$ a formula. We assume that (a) $\mathscr{M}, c_{i}, w \vDash_{2.2} K_{j} \phi$, and, ad absurdum, that (b) $\mathscr{M}, c_{i}, w \nvdash_{2.2} K_{j} K_{j} \phi$. From (a), it follows that for any $w^{\prime}$, if $\mathscr{K}_{j}^{j} w w^{\prime}$ then $\mathscr{M}, c_{j}, w \vDash_{2.2} \phi$. From (b), it follows that there are two worlds $w_{1}, w_{2}$, s.t. $\mathscr{K}_{j}^{j} w w_{1}, \mathscr{K}_{j}^{j} w_{1} w_{2}$, and (c) $\mathscr{M}, c_{j}, w_{2} \not \nvdash_{2.2} \phi$. Since the relations are supposed to be transitive, we have $\mathscr{K}_{j}^{j} w w_{2}$; and therefore by (a), we get $\mathscr{M}, c_{j}, w_{2} \vDash_{2.2} \phi$, which directly contradicts (c). Conclusion: $\mathscr{M}, c_{i}, w \vDash_{2.2} K_{j} \phi \rightarrow K_{j} K_{j} \phi$.

(1.2): $\quad$ Let $\mathscr{M}$ be a contextual model with transitive relations, $c_{i}$ and $c_{j}$ two contexts s.t. $\mathscr{R}_{c_{j}} \subseteq \mathscr{R}_{c_{i}}, w$ a world, and $\phi$ a formula. We assume that (a) $\mathscr{M}, c_{i}, w \vDash_{1.2}$ $K_{j} \phi$, and ad absurdum that (b) $\mathscr{M}, c_{i}, w \not \not_{1.2} K_{j} K_{j} \phi$. From (a), it follows that for all $w^{\prime}$, if $\mathscr{K}_{j}^{i} w w^{\prime}$ then $\mathscr{M}, c_{j}, w \vDash_{1.2} \phi$. From (b), it follows that there are two worlds $w_{1}, w_{2}$ s.t. $\mathscr{K}_{j}^{i} w w_{1}, \mathscr{K}_{j}^{j} w_{1} w_{2}$, and (c) $\mathscr{M}, c_{j}, w_{2} \not \not_{1.2} \phi$. Since $\mathscr{R}_{c_{j}} \subseteq \mathscr{R}_{c_{i}}$, we may infer $\mathscr{K}_{j}^{i} w_{1} w_{2}$ from $\mathscr{K}_{j}^{j} w_{1} w_{2}$. Now, since the relations are supposed to be transitive, from $\mathscr{K}_{j}^{i} w w_{1}$ and $\mathscr{K}_{j}^{i} w_{1} w_{2}$ we may infer $\mathscr{K}_{j}^{i} w_{w} w_{2}$; therefore, by (a), we have $\mathscr{M}, c_{j}, w_{2} \vDash_{1.2} \phi$, which contradicts (c). Conclusion: $\mathscr{M}, c_{i}, w \vDash_{1.2} K_{j} \phi \rightarrow K_{j} K_{j} \phi$.

\section{Proposition 8 Validity of (5):}

- Schema (5) is (2.2)-valid in contextual models $\mathscr{M}$ with Euclidian $K_{j}$ and $\mathscr{R}_{c_{k}}$ relations;

- Schema (5) is not (1.2)-valid in contextual models $\mathscr{M}$ with Euclidian $K_{j}$ and $\mathscr{R}_{c_{k}}$ relations. However, in such models the following instantiation of (5) holds: $\mathscr{M}, c, w \vDash_{1.2} \neg K_{j} \phi \rightarrow K_{j} \neg K_{j} \phi$ if $\mathscr{R}_{c_{j}} \subseteq \mathscr{R}_{c_{i}}$.

Proof.

The proof is similar to that for (4). We assume that we both have $\mathscr{M}, c_{i}, w \vDash_{-.2} \neg K_{j} \phi$ and $\mathscr{M}, c_{i}, w \vDash_{-.2} \neg K_{j} \neg K_{j} \phi$, which leads to contradictory requirements on a world $w_{0}$, the first assumption requiring that $\mathscr{M}, c_{j}, w_{0} \not \nvdash_{-.2} \phi$, the second implying that $\mathscr{M}, c_{j}, w_{0} \not \nvdash_{-.2} \phi$ given the Euclidianity of $\mathscr{K}_{j}^{j}$ for (2.2), and that of $\mathscr{K}_{j}^{i}$ for (1.2).

Proposition 9 Definition (1.1) with w-constant relevance function $\mathscr{R}$, i.e. s.t. for any context $c, \mathscr{R}(c)$ is constant, reduces to a case of standard Kripkean semantics.

Proof: Suppose the relevance function $\mathscr{R}$ in a contextual model $\mathscr{M}=\left\langle W,\left\{\mathscr{K}_{j}: j \in I\right\}, \mathscr{C}, \mathscr{R}, V\right\rangle$ is $w$-constant, and consider a particular context $c_{i}$. We build a standard Kripke model from $\mathscr{M}, \mathscr{M}^{c_{i}}=$ $\left\langle W^{c_{i}},\left\{\mathscr{K}_{j}^{c_{i}}: j \in I\right\}, \mathscr{C}^{c_{i}}, \mathscr{R}^{c_{i}}, V\right\rangle$, where $W^{c_{i}}=\mathscr{R}\left(c_{i}\right)$, and $\mathscr{K}_{j}^{c_{i}}$ is the restriction of $\mathscr{K}_{j}$ to $\mathscr{R}\left(c_{i}\right)$. Then, for any world $w \in \mathscr{R}\left(c_{i}\right)$ :

if $\alpha$ is an atom: $\mathscr{M}, c_{i}, w \vDash_{1.1} \alpha \Leftrightarrow w \in V(\alpha) \Leftrightarrow \mathscr{M}^{c_{i}}, w \vDash \alpha$ 
For points (ii) and (iv) below, we accept the recurrence hypothesis: $\mathscr{M}, q, w \vDash_{1.1} \varphi \Leftrightarrow \mathscr{M}^{c_{i}}, w \vDash \varphi$, and for point (iii), the corresponding hypotheses with $\varphi_{1}$ and $\varphi_{2}$.

(ii)

$$
\mathscr{M}, c_{i}, w \vDash_{1.1} \neg \varphi \Leftrightarrow \mathscr{M}, c_{i}, w \not \nvdash_{1.1} \varphi \Leftrightarrow \mathscr{M}^{c_{i}}, w \not \models \varphi \Leftrightarrow \mathscr{M}^{c_{i}}, w \vDash \neg \varphi
$$

(iii) $\quad \mathscr{M}, c_{i}, w \vDash_{1.1} \varphi_{1} \vee \varphi_{2} \Leftrightarrow \mathscr{M}, c_{i}, w \vDash_{1.1} \varphi_{1}$ or $\mathscr{M}, c_{i}, w \vDash_{1.1} \varphi_{2} \Leftrightarrow \mathscr{M}^{c_{i}}, w \vDash \varphi_{1}$ or $\mathscr{M}^{c_{i}}, w \vDash$ $\varphi_{2} \Leftrightarrow \mathscr{M}^{c_{i}}, w \vDash \varphi_{1} \vee \varphi_{2}$

(iv) $\quad \mathscr{M}, c_{i}, w \vDash_{1.1} K_{j} \varphi \Leftrightarrow$ for all $w^{\prime} \in W$ s.t. $\mathscr{K}_{j} w w^{\prime}$, if $w^{\prime} \in \mathscr{R}\left(c_{i}\right)\left[=W^{c_{i}}\right]$ then $\mathscr{M}, c_{i}, w^{\prime} \vDash_{1.1}$ $\varphi \Leftrightarrow$ for all $w^{\prime} \in W^{c_{i}}$ s.t. $\mathscr{K}_{j} w w^{\prime}, \mathscr{M}^{c_{i}}, w^{\prime} \vDash \varphi \Leftrightarrow \mathscr{M}^{c_{i}}, w \vDash K_{j} \varphi$. 